\title{
Erinnerte "Wirklichkeit" and erzählte "Fiktion": Das Neuseeland der Otti Binswanger in den Jahren 1939-1948
}

Otti und Paul Binswanger kamen 1939 nach Neuseeland. Auch sie hatten; wie Wolfskehl, zuvor in Italien gelebt und mußten als "Fremde 'nichtarischer Abstammung' binnen sechs Monaten das Land verlassen." 1 Otti hatte eine Cousine auf der Süd-Insel Neuseelands, die mit einem Neuseeländer verheiratet war; diese verwandtschaftliche Beziehung ermöglichte das so lebensnotwendige Einreisevisum. Die ersten Wochen nach ihrer Ankunft verbrachten die Binswangers auf der Farm ihrer Verwandten, um sich später in Christchurch anzusiedeln, in der Hoffnung auf eine Anstellung Pauls als Romanist an der Universität. Diese Hoffnung zerschlug sich bald. Um ihr Leben zu fristen, gab Paul private Deutschstunden und Otti versuchte sich, erfolgreich, an vielem, u. a. auch am Schreiben von Erzählungen. Ihr Band mit dem Titel "AND HOW DO YOU LIKE THIS COUNTRY?" erschien 1945 by Walter Brookes in Christchurch. Nelson Wattie nimmt 1996 auf diesen Erzählband Bezug und berichtet über das Erstauntsein der Herausgeberin von Kite 1992 als sie im Zusammenhang mit "vergessenen Schätzen der neuseeländischen Literatur" entdeckte, daß die Autorin des Erzählbandes ein Flüchtling war. Wattie schreibt: "So puzzled was our editor by the volume that she "suspected a hoax by Glover or Fairburn' and she treated it with a certain ambivalence, apparently hesitating between respect for the assumed refugee origins of its author and scepticism about its literary worth."3

Als mir Friedrich Voit vor einiger Zeit diesen Band zum Lesen gab, war ich zunächst äußerst belustigt darüber, daß eine Deutsche, die erst sechs Jahre im Land verbracht hat, mit dem Anspruch auftritt, daß man ihre Erzählungen nicht als die einer Außenseiterin lesen sollte, sondern durchaus als neuseeländische Literatur. Sie schreibt im Vorwort:

Author of these New Zealand stories, I have been, until some time before the war, a foreigner to this country. And yet I dare to present them not as stories of a New Zealand such as some presumptuous

1 „Der Albatros“, unveröffentlichtes Manuskript, S. 156.

2 Newsletter of the Association of New Zealand Literature.

3 Kite 10, S. 8. 
intruder may see from outside. They are in fact inside stories.

Nachdem ich Otti Binswangers unveröffentlichte Autobiographie gelesen und besondere Aufmerksamkeit den Kapiteln über Neuseeland gewidmet hatte, verglich ich ihre Beobachtungen, Gefühle und kritischen Bemerkungen mit ihren fiktionalen Texten. Ich fand die Erzählungen anmaßend. Nicht wegen ihres sprachlichen Anspruches als englischsprachige Literatur zu gelten; dieser Anspruch hat mich eher beeindruckt. Es war vielmehr die Erzählperspektive, die mir anmaßend vorkam. Sie erhebt den Anspruch auf eine imaginierte Omnipotenz, die sich zumeist in der Erzählform der Erlebten Rede zur Schau stellt. Sicherlich sollte man Otti zugute halten, daß diese Erzählungen autodidaktische literarische Versuche waren, die sich unter die anderen Versuche, zum Lebensunterhalt beizutragen, einordnen ließen. Vergleicht man jedoch die Haltung der Erzählerin von "fiktiven" Texten mit der der Autobiographin, ergibt sich eine erstaunliche Ähnlichkeit. Bei der Begegnung mit dem Fremdartigen werden die eigenen soziokulturellen Kategorien als Bewertungsmaßstäbe verwendet, ohne die Arbiträrität solcher diskursiven Selbstverständlichkeit zu thematisieren. Somit ist die Grundstruktur in der Konstruktion der erinnerten "Wirklichkeit" und der erzählten "Fiktion" eine gleiche. Sie ist eine Interpretation des Fremden aus einer als unproblematisch dargestellten Perspektive, die sich ihres kulturspezifischen und daher arbiträren Charakters nur selten bewußt ist.

Ich hatte ursprünglich angenommen, daß die Erzählungen eine idealisierte Welt darstellten im Vergleich zu der in der Autobiographie erinnerten, waren sie doch an ein jeweils recht unterschiedliches Publikum gerichtet. Doch als ich Anfang dieses Jahres Autobiographie und Erzählband vergleichend las, kam ich zur Einsicht, daß dies überhaupt nicht der Fall war. Vielmehr schien Otti Binswanger versucht $\mathrm{zu}$ haben, zu verdichten, was ihr am problematischsten an der neuseeländischen sozialen Kultur erschienen war. (Dies ist wiederum meine Interpretation.)

Binswagers verließen 1948 Neuseeland. Paul starb 1961 in Italien, und Otti schrieb ihre autobiographischen Aufzeichnungen mit dem Titel „Der Albatros“ (auf deutsch natürlich) ein Jahr später, d. h 17 Jahre nach den englischsprachigen Erzählungen. Jene erinnerte "Wirklichkeit" des Lebens in Neuseeland, worauf ich mich hier beschränke, geht auf eine Zeit zwanzig Jahren früher zurück. Ich gehe von der Annahme aus, daß die Verfasserin die ihr am wesentlichsten erscheinenden Eindrücke und Ereignisse ihrer in Neusee- 
land verbrachten neun Jahre festzuhalten suchte. Die Frage, die ich mir nun stellte war, wie das Erinnerte sich mit den "Insider stories" vergleichen ließe; gab es Überschneidungen oder war die Perspektive der beiden Texte marktspezifisch und daher notwendigerweise anders? Notwendigerweise?

Ich erlebte eine Überraschung. Ich fand, daß Ottis Ansichten über die Begegnung mit der neuseeländischen Variante der hauptsächlich angelsächsischen Lebensführung und Einstellung beiden Textund Sprachformen zu Grunde lagen. Ich würde sogar behaupten, daß ihre literarische Produktion Zeichen einer utopischen Subversion trägt. Was ich damit meine, ist das folgende: durch den Anspruch, daß ihre Erzählungen neuseeländische Literatur repräsentieren, wird eine Kritik an spezifischen Verhältnissen und soziokulturellen Eigenartigkeiten als eine Neuseeland-interne Kritik vorgegeben. Dadurch sollte dieser Kritik Authentizität und Gewicht verliehen werden. Diese, ihr meinerseits unterstellte, Absicht der Autorin muß keine bewußte gewesen sein.

Was mich eigentlich an Otti Binswangers Darstellung eines erinnerten und fiktionalen Neuseelands interessiert, ist die vollkommene Abwesenheit eines Bewußtseins des Arbiträren der eigenen Bewertungskategorien. Mit anderen Worten: Die Verfasserin glaubt durchaus an universelle Wertmaßstäbe, in deren Besitz sie ihre Umgebung kritisch einstufen kann. Eine Alterität, die das eigene Selbstverständnis bedroht und eine Entfremdung des Subjekts von seiner sozialen Umgebung verursacht, wird als veränderungsbedürftig empfunden. Meine Einstellung war ursprünglich eine ähnliche, obwohl ich erst im Jahre 1971 in dieses Land kam. Noch heute kann ich mich sehr wohl mit Ottis Reaktionen auf ihre soziale Umwelt identifizieren. Immerhin bin ich um die Einsicht reicher geworden, daß eine solche Haltung in eine psychische Sackgasse führt. Da eine völlige Angleichung an die als selbstentfremdend empfundenen soziokulturellen Praktiken kaum möglich und zumindest problematisch ist, scheint eine Einsicht in die historischen Gründe der Andersartigkeit das Unbehagen zu vermindern, das durch abgelehnte Kulturlandschaften oder Begegnungen hervorgerufen wird.

Otti Binswanger versucht in ihren Erinnerungen geschichtliche Kenntnisse über die neuseeländischen Städtebauer als Begründung ihrer Sicht von Timaru z. B. einfließen zu lassen. Sie beschreibt ihren ersten Eindruck von Timaru wie folgt: 
Timaru liegt am Meer, eine prosperierende, wachsende Stadt schön ist sie darum nicht. Ihre niedrigen Holzhäuser, Wellblechdächer und Landstrassen trugen immer noch den Stempel des Provisoriums, des "wir-können-jetzt-nicht-anders". Ihre Gründer, sie kamen so unendlich weit her, fromme, tapfere Menschen, sie klammerten sich an die strengen Traditionen [Hervorhebung von LKW] von ihrer Heimat und gaben sie um nichts preis. Sie waren weder Entdecker noch Abenteurer, sie waren Ladenbesitzer, enttäuschte Geschäftsleute, landlose Farmer, die sich in kirchlichen Gruppen zusammengeschlossen hatten. Ihre Arbeitskraft und wohl auch ihr Mut hat sie zu guten Farmern und Geschäftsleuten gemacht. Ihre kleinbürgerliche, provinzielle Begrenztheit machte sie nicht zu Städtebauern - die Musen waren ihnen nicht hold. ${ }^{4}$

Die 'strengen Traditionen,' an die sich Ottis neuseeländische Zeitgenossen, jene Abkömmlinge der ehemaligen Kolonialisten, 'klammerten,' erschienen der Europäerin, die nicht nur das Städteleben von Berlin und München kannte, aber auch aktiv an alternativen Bewegungen zu Anfang des Jahrhunderts teilgenommen hatte, wie im "Jungwandervogel" und der "Siedlung Frankenfeld", provinziell und phantasielos beengend. Nelson Wattie kommentiert in Hinblick auf Ottis Vergangenheit in der Wandervogel-Bewegung und den Schwierigkeiten einer solchen Frau mit den als rückständig empfundenen soziokulturellen Gegebenheiten Neuseelands zur Zeit ihrer Emigration wie folgt: "By participating in this movement [er bezieht sich auf den Wandervogel], Otti was one of many thousands of young people who were looking for values different from those of their parents but often finding themselves in tightly organised structures which had a whiff of authoritarianism about them." 5

Eine solche autoritäre Struktur ist für Otti das neuseeländische Schulwesen gewesen. Als Gymnastiklehrerin fand sie Anstellung u. a. auch an Schulen. Sie erinnert sich:

Weniger erfolgreich und sinnvoll war meine Arbeit mit den Schul-
mädchen. Sie blickten unsicher und misstrauisch aus ihrer um-
mauerten Schülerwelt auf mich, ich stand ihnen sehr fern. Die Leh-
rerautorität, der sie kritiklos gehorchten, untergrub ich wo ich
konnte. Vergebens versuchte ich, als Kamerad mit ihnen zu
arbeiten, sie verstanden mich nicht. Ich hatte nicht die geringste
Absicht, eine Schoolmistress darzustellen. Als sie einmal über ihre
steifen Arme klagten, weil sie am Vortag fünf Minuten mit ge-
streckten Armen hatten stehen müssen, da ihre Turnlehrerin ans

4 „Der Albatros“, S. 176-177.

5 Kite 10, S. 9. 
Telefon gerufen worden war und vergessen hatte, den Befehl zum Fallenlassen der gequälten Glieder zu geben, verging mir das Lachen. Ich verlor die Lust und gab die Arbeit auf. 6

Vergleichsweise fand Otti, daß die Maori Schulmädchen ihren Bemühungen gegenüber empfänglicher waren. Sie schreibt über ihre Arbeit an einem Internat wie folgt:

Man hatte mir auch den Unterricht der Maori-Mädchen anvertraut, die in einem Internat zu guten kleinen englischen Hausfrauen erzogen wurden. Sie waren Südseeinsulaner, Polynesier und also mit vierzehn Jahren oder auch früher ausgewachsene kleine Frauen. Auch sie wurden durch Schuluniformen verhässlicht und sie wurden genau so gehalten wie die vierzehnjährigen, fischblütigen englischen Kinder aus den kalten Zonen. Ich habe alles versucht, um die Urschönheit ihrer polynesischen Abkunft in ihnen aufzuwecken, ihr Lachen, ihre Freudefähigkeit. Am Ende haben sie mich geliebt und in ihrem Laufen und Springen konnte man sie wieder als die Naturkinder warmer Inselreiche erkennen. ${ }^{7}$

Interessanterweise thematisiert Otti diese Erfahrungen mit den Maori Mädchen in keiner der Erzählungen. Sie wählt vielmehr einen Jungen zum Protagonisten ihrer Geschichte "So green was my playground". Es ist eine Skizze der Entwicklung eines naturnahen und selbstgenügsamen Knaben, der durch das Schulsystem zum perfekten Produkt einer vorprogrammierten Ideologie des ferngesteuerten Massenmenschen wird, zum Typ des sportbegeistērtèn, anti-intellektuellen, freundlich beschränkten Neuseeländers. Der Text ist teils als Satire geschrieben, teils als psychologische Studie der Ängste, die der kleine Junge ausstehen muß, bevor er den Mechanismus der Anpassung lernt. Anpassung an Dinge und Werte, die ihm wesensfremd sind. Wie korrespondiert nun diese Studie eines vorprogrammierten Automaten mit Ottis Erinnerungen an das neuseeländische Schulwesen in ihrer Autobiographie? Sie schreibt:

Ein weiterer Schock war für mich der Anblick der in verhässlichende Uniformen gesteckten Schuljugend. Vielleicht waren die Schulen gut und gründlich - ich sah nur die Reihen von schwarzbestrumpften, filzhutbedeckten, krawattentragenden Kindern und Halbwüchsigen und schauderte, schauderte doppelt als ich erfuhr,

6 ,Der Albatros", S. 187-188.

7 Ebd., S. 188. 
dass man in den Schulen die Kinder noch prügelte - war ich auf einem anderen Planeten angelangt? "Das macht doch nichts, die Kinder ziehen das Geprügeltwerden dem Nachsitzen vor" - wie gut, dass ich keine Kinder in die Schule zu schicken hatte. - Die Kinder wurden auf die Hände geschlagen, die Jungen bekamen Schläge mit dem Rohrstock. Beim Direktor anstellen zur Exekution. Der Junge legt sich über den Stuhl, der Direktor drischt und zählt und schreibt die ausgeteilten Hiebe in ein Buch ein. Der Junge richtet sich auf und sagt "thank you, Sir". Ich hätte meine Jungen angehalten, den Direktor in den Bauch zu boxen und hätte ihnen hierfür den nötigen Unterricht erteilen lassen. "The Corporal Punishment" oder das Eingeständnis pädagogischer Unzulänglichkeit und Phantasielosigkeit ist heute noch in britischen Schulen zu finden. Ein junger deutscher Emigrant unterrichtete an einer Elementarschule. Die kleinen Jungen beschwerten sich bei ihm, weil er nicht schlug. Er fand dann allerdings heraus, dass dem einzelnen nicht so viel dran lag, selber geschlagen zu werden, aber dass sie zusehen wollten, wie der Lehrer die anderen prügelte. Die Macht der Schule ist gross und reicht weit über die Schulmauern und Schulzeiten hinweg. ${ }^{8}$

Nach Ottis Auffassung werden die Kinder "früh eingereiht in dieses Schema, das man hier mit 'Freiheit' bezeichnet, in dem sie alle gut und freundlich leben, nicht ahnend, dass es in Wahrheit die Angst vor der Freiheit ist, aus der man dies Schema entwickelt hat."9 Diese "Angst vor der Freiheit" als dominante Ideologie der neuseeländischen Lebensführung, die in dem von ihr skizzierten Schulsystem vorgeprägt scheint, gibt Otti vor, auch in der Anlage der Stadt Christchurch zu erkennen. Mit ihren geordneten, aber abgesteckten Gärten und Grünflächen, reflektiere sie jene Angst vor der Freiheit, die Otti so beengend fand und in ihrer Tätigkeit als Gymnastiklehrerin zu unterwandern suchte. Sie hoffte, daß sie durch ihre Arbeit mit Menschen, die außerhalb jener dominanten Ideologie, "des englischen verdrängenden Schweigens", angesiedelt waren, mehr Erfolg erzielen könne. In ihren eigenen Worten: "Mein Begehren nach einer Freiheit ohne Angst mag es gewesen sein, die mich veranlaßte keine Ruhe zu geben, bis ich mit meiner Arbeit in 'Sunnyside' (der 'Sonnenseite') Einlaß fand, der Irrenanstalt von Christchurch."10

Dieser Versuch Ottis, mit Menschen in Kontakt zu kommen, denen man die äußere Freiheit aberkannt hat, die sich aber von dem verinnerlichten Diktat der Normativität befreit hatten, läßt verschiedene

8 Ebd., S. 183-184.

9 S. 194.

10 Ebd., S. 195. 
Interpretationen zu. Man könnte in ihm eine völlige Ablehnung der als restriktiv empfundenen, neuseeländischen öffentlichen Diskurse sehen, deren als repressiv erlebten Autorität Otti zu entkommen strebte. Was aber könnte die erfahrungsbedingte oder ideologisch/philosophische Grundlage von Ottis Einstellung gewesen sein, die sie dazu veranlaßte, auf eine "Freiheit ohne Angst" unter Menschen zu hoffen, für die die Gesetze der symbolischen Ordnung ihre Gültigkeit verloren haben und von denen manche im Zwischenland zwischen einer gesellschaftlich sanktionierten realen und einer imaginierten Wirklichkeit angesiedelt waren. Das bürgerlich liberale, intellektuell und wissenschaftlich experimentierfreudige Elternhaus, die Berührung mit Ausläufern der Lebensreformbewegung Anfang des 20. Jahrhunderts, - die auch zur ersten Bekanntschaft mit Wolfskehl führte, die relative Freiheit, mit der Otti zur Zeit ihrer beruflichen Tätigkeit ihren Arbeitsplatz wechselte, mögen dazu beigetragen haben, das Erbe deutschen idealistischen Gedankengutes als auf die fremde Wirklichkeit anwendbar $\mathrm{zu}$ glauben. Ihrer Freiheitskonzeption mußte jenes Vertrauen auf die gesellschaftlich befreiende Funktion der Vorstellungskraft zugrunde gelegen haben, die Herbert Marcuse in seiner sozialpsychologischen Utopie, Eros and Civilisation ${ }^{11}$ zehn Jahre später, 1955, philosophiegeschichtlich fundiert und die amerikanische und westeuropäische Jugend der 60er Jahre inspirierend, ausgeführt hatte. Man sei freilich auch auf die Schriften Anfang der 70er Jahre von Laing, Cooper und Szász hingewiesen.

Otti wurde während des Ersten Weltkrieges "Schülerin der 'Sozialen Frauenschule', die, [wie sie in „Albatros“ schreibt] Alice Salomon in Berlin gegründet hatte und leitete.

[Die] Lehrkräfte trugen die grossen Namen der deutschen Frauen bewegung. Sie verstanden es, [erinnert sich Otti] uns die Sicht zu öffnen für soziale Probleme und ihre theoretischen und praktischen Lösungen. Sie vermittelten auch den geistigen Hintergrund, der jene Lösungen ermächtigte. Dieser Schule danke ich den ersten Hinweis auf Kants und Schillers philosophische Schriften. Zu meiner eigenen Überraschung wurden sie zur wichtigsten Anregung meiner Ausbildung."12

Genaueres sagt Otti darüber nicht aus. Ich vermute, daß es um die Funktion von Vorstellungskraft und Ästhetik bei Kant und Schiller

11 Marcuse, Herbert, Triebstruktur und Gesellschaft, Frankfurt a. M. 1965.

12 „Der Albatros“, S. 58-59. 
ging, deren Relevanz Otti, unter der Leitung ihrer feministischen Lehrerinnen, für ihre eigene Weltsicht sowie auch für ihre spätere Arbeit nutzbar zu machen suchte. Worum es zu gehen scheint, entspricht der Interpretation von Marcuse. Er schreibt:

\begin{abstract}
Die Geschichte des Ausdrucks "Ästhetik" in der Philosophie spiegelt die repressive Behandlung der sinnlichen (und damit "körperwirklichen ") erkennenden Prozesse wider. [...] In die Kulturphilosophie eingeführt, zielt der Begriff auf eine Befreiung der Sinne hin, die keineswegs eine Zerstörung der Kultur zur Folge hätte, sondern ihr eine festere Grundlage und sehr erweiterte Möglichkeiten verschaffte. Durch einen Grundantrieb wirkend - nämlich den Spieltrieb - würde die ästhetische Funktion "auch alle Nötigung aufheben, und den Menschen sowohl physisch als moralisch in die Freiheit setzen".13
\end{abstract}

Otti wurde zunächst Sozialpädagogin, arbeitete in Kindergärten, widmete sich sozial benachteiligten Kindern. Enttäuscht von ihrer eigenen Machtlosigkeit, gab sie diese Arbeit auf und wirkte für einen Jungendklub, einen Verlag $\mathbf{u}$. a. und mischte sich unter die verschiedensten Vereinigungen und Bewegungen. So machte sie auch die Bekanntschaft von Marie Buchhold, die um Mitglieder für ihre Jungendsiedlung Frankenfeld bei Gernsheim warb. 1920 zog Otti auf die Siedlung. Wieweit sie hier mit Idee und Praxis der rhytmischen Gymnastik in Berührung gekommen ist, läßt sich schwer sagen. Otti nimmt lediglich im "Albatros" Bezug auf den Besuch einer Mädchengruppe von der "Gymnastikschule Loheland in der Rhön", über die sie eher ablehnend urteilt: "Sehr gut gewachsen schritten sie rhytmisch daher, aller Impulse bar, eisgekühlt-ausbalancierte Vestalinnen, hoffend, die Gnade der Muse zu ertanzen, zu erwandeln und asketisch zu erhungern."14 Obwohl Otti kurze Zeit darauf selbst einen Atemgymnastik-Kurs absolvierte und von da an ihr Geld vor allem als Gymnastiklehrerin verdiente, auch in Neuseeland, nimmt sie eine kritische Haltung gegenüber einen großen Teil der mit einer Ideologie verbundenen Gymnastikpraxis ein, die, wie sie schreibt, in Deutschland in den 20er Jahren zu einer Bewegung geworden war. Sie schreibt:

"Körperkultur", Körperbewusstsein", "Körper-Seele”. Neue Werte, neue Begriffe, neue Taten. Gymnastiksysteme und Gymnastikschulen entstanden in Mengen, und da dies ja in Deutschland stattfand,

13 Marcuse, Triebstruktur und Gesellschaft, S. 180.

14 „Der Albatros“, S. 84. 
verbanden sich die Systeme wiederum fest mit bestimmten Weltanschauungen und Ernährungsprinzipien. Es gab sicher große Persönlichkeiten unter den Gründern einzelner Schulen, deren grundlegende Erkenntnisse für ihre Zeit und über diese hinaus von Bedeutung gewesen sind. [Doch e]in großer Teil dieser Gymnastik-Sucht war Kitsch oder der Mode zuzuschreiben, die für Mütter und Frauen über vierzig Knabenfiguren vorschrieb. 15

Auch bemängelt Otti an diesen Gymnastik-Praktiken eine Spontaneität, die für sie so wichtig und Voraussetzung von Kreativität und Freiheit wird. Wenn man nun Spontaneität mit Raum für Phantasie übersetzt, so läßt sich die Verbindung zu Otti Binswangers eigener Lebensphilosophie finden, für die sie entscheidende Impulse in der oben erwähnten Sozialschule Alice Salomons erhalten hatte. Was sie versuchen wollte, war, in der Gymnastik auch Raum für die Phantasie als Selbstbefreiung zu bewahren. Zugleich wollte sie die Anwendung einer solchen Gymnastik mit der eigenen sozialen Verantwortung verbinden. Also doch eine Art Sendungsbewußtsein? Sie notiert im „Albatros“:

Durch einen Glücksfall konnte ich einen Kurs für Atemgymnastik bei Schlaffhorst-Andersen in Rothenburg an der Fulda mitmachen, der mich sehr anregte und in mir eine neue Berufsidee weckte. Ich wusste einiges vom menschlichen Körper und begann, die hier erworbenen Kenntnisse und Erfahrungen in eigener Arbeit auf Muskelspannung und Entspannung in der Bewegung zu übertragen. Meine Aufmerksamkeit galt den Benachteiligten, die durch Haltungs- oder Arbeitsschäden hässlich Gewordenen und den Alternden. ${ }^{16}$

Um also auf Neuseeland zurückzukommen: Nach ihren fehlgeschlagenen Versuchen, in Schulen das ihr vorschwebende Ziel zu erlangen, hoffte Otti durch ihre Arbeit in der Nervenheilanstalt auf Menschen zu treffen, die sich der Ängste der Freiheit entledigt hatten und von der Produktivität der Phantasie repressionsfreien Gebrauch machen würden. Sie schien solche Menschen gefunden zu haben, denn sie bemerkt über ihre Zeit in Sunnyside:

Hier hatte ich gute Freunde - die auf der Grenze zwischen dem Licht und der Dunkelheit leben, erhaschen zuzeiten von der Helle einen kurzen Glanz, der auf kurze Sekunden die Geheimnisse des Lebens

15 Ebd., S. 99.

16 Ebd., S. 100. 
mit einer Kraft anstrahlt, wie sie nur wenigen zuteil wird. Frei aller Bürde schauen sie mit letzter Deutlichkeit Bewegungen des Daseins, seine Schönheit, seine Trauer, sein Verlöschen, weil sie des Mutes nicht mehr bedürfen. - In solchen Momenten konnte ich angstlose Freiheit wittern. ${ }^{17}$

Otti versuchte, durch Musik und einfache Gymnastik-Übungen, Anregung und Freude in das von ihr als chaotisch empfundene Dasein der Insaßen in der Frauenabteilung für "Unheilbare" zu bringen. Sie beobachtete den günstigen Effekt ihrer Bemühungen auf die Frauen und versuchte, die Ärzte darauf aufmerksam zu machen. Man begegnete ihr mit wohlwollend skeptischem Lächeln, aber es blieb alles beim Alten. Die Ärzte waren, wie Otti berichtet, aus Mangel an Personal überfordert und desinteressiert an unkonventionellen Heilungstherapien.

Was Otti in Sunnyside beobachtet hatte, machte auf sie einen nachhaltigen Eindruck, denn sie widmet in ihrer Autobiographie einige kritische Seiten dieser Institution und der allgemeinen Haltung in der (weißen) Bevölkerung den Nervenkranken gegenüber. Einer der eindruckvollsten ihrer fiktionalen Texte mit dem Titel "Forgotten" ist eine einfühlende Studie in das imaginierte Los einer jungen Frau, die durch ihre besondere Haltung von den anderen Schicksalsgefährtinnen absticht. Der Text beginnt mit einer Schilderung, die sich auf eine knappere, aber im Wesentlichen kaum abweichenden Form, in den siebzehn Jahre später verfaßten deutschsprachigen autobiographischen Erinnerungen wiederholt.

THE LONG WIDE HALL is poorly lit. The large blazing fire at its far end spreads more light than the few bulbs dangling from the ceiling. Under one of these, just where the draught from the opposite doors and an open window sweeps past like a frosty wind, a woman stands on a shaky chair. In her uplifted hands she holds some printed sheets. Thus she tries to read and to catch sufficient light from the meanly rationed electricity. She is tall and slender, and with a sort of hood tightly drawn over a well shaped head, in her tense erectness, with her raised arms that seem not to feel the effort of her posture, she resembles a stonefigure on one of the Gothic cathedrals, steady and obedient to her call in eternal loneliness. A kind of patina lies over her features which seem without age, noble and childlike, withered under an unknown fate. Here she stands, on the chair, under the faint light, as she has stood

17 Ebd., S. 197. 
before, during all this hour of the wintry day, while most of the other inmates are crowding in front of the glaring heat of the fire. At first she really tried to read and climbed on the chair to get close to the light. But the printed words hardly reach her now. She has found the isolation she was longing for, where others could not touch her. There is no one near on that higher plane, the hostile noises and parading shadows have become immeasurably distant. 18

In der Autobiographie liest sich die betreffende Stelle folgendermaßen:

Und diese junge, schlanke Frau stand immer, wenn ich kam, auf einem Stuhl unter dem kümmerlichen Licht einer elektrischen Birne und las. Sie trug eine enge Kappe über den Haaren, hielt das Buch in beiden Händen, den Kopf kaum geneigt. Sie war sehr schön. Ihr langer, anliegender Rock verriet ihre schlanken Schenkel und Beine, sie glich einer Steinfigur an einem gotischen Dom. Um sie war eine unabwendbare Entrücktheit eines Märtyrers. Die Ruhe und Beherrschtheit ihrer Haltung hätte wohl einem Arzt einen Weg zur Heilung zeigen können, doch solche Ärzte gab es hier nicht. Und niemand fand sich, der ihr ein besseres Licht gab. ${ }^{19}$

Eine anderes Stück aus dem Erzählungsband trägt den Titel "Turnips" [Kohlrüben]. Es ist eine Momentaufnahme vom Anfang des Krieges, wie die neuseeländischen Nachbarn eines Flüchtlingehepaares und die betroffene Ehefrau selbst ihn durch die Reaktionen ihrer Nachbarn erlebt. Die Nachbarn reagieren vorwiegend [von gegenüber, von links und von hinten] mitfühlend und solidarisch; allein der Nachbar von "rechts" wird als fremdenfeindlich beschrieben. Seine Einstellung entspricht jenen Vorurteilen, die in einer Schicht der Bevölkerung zu jener Zeit gang und gäbe waren. Ann Beaglehole widmet das erste Kapitel ihres Buches A Small Price to Pay ${ }^{20}$ einer kritischen Übersicht der diesbezüglichen Dokumente und untersucht die Gründe der Fremden- und Judenfeindlichkeit in den maßgeblichen Behörden sowie gewissen Schichten der Bevölkerung. In Otti Binswangers Text wird der Nachbar von rechts zum Repräsentant eines solchen fremdenfeindlichen Diskurses, für den sie die Vorlage in Zeitungsartikeln, Leserbriefen, Verordnungen und Gespächen gefunden haben muß:

18 Binswanger, And How Do You Like This Country?, S. 21.

19 „Der Albatros“, S. 196.

20 Beaglehole, Ann, A Small Price to Pay, Wellington, 1988 
Who knows why they came here, with the whole globe to choose from, just to us. Couldn't they have gone somewhere else? Isn't the world big enough, are not other countries larger and better for foreigners? Why must we have them all? Who can prove that they were persecuted? And if they were, there was probably a good reason for it. There is a lot of eyewash and propaganda in all this about them being driven away under threats to their lives. And even if it was true, here they are foreigners, and we have no time for foreigners in war time .... But they will now intern, thank God, all these foreigners who are taking decent peoples jobs and money. The government will have to act, and no doubt they will. If they are not interned by next week he will write a letter to the editor of the local paper and will sign it "Intern The Lot;" that will be fine. 21

Seine Frau findet es aufregend, daß die Nachbarn, mit ihren seltsamen Sitten, wohl bald von der Polizei abgeholt werden: "she goes from time to time to the window to see if the car which will round them up has already arrived. She is very excited and feels right in her whole system that there is a war on." 22

Die anderen Nachbarn hingegen geben ihrem Mitgefühl der "Frau mit dem fremden Namen" gegenüber Ausdruck, indem sie ihr als nützliches Geschenk Kohlrüben bringen und über den Zaun werfen. Diese Kohlrüben, als Symbol des guten Willens von der "Frau mit dem fremden Namen" erkannt, lassen Assoziationen an den Ersten Weltkrieg in Deutschland entstehen. Angenehmere Erinnerungen erweckt ein Glas Quittenmarmalade, das durch ein Loch in dem Zaun von einer Hand in ihren Garten geschoben wird. Es erinnert sie an den Quittenbaum in ihrem früherem Zuhause. Otti endet ihren Text mit der Einsicht der Fremden:

The turnips are on the verandah, as a memorial to the first good fellowship in the foreign land, and when she rushes past to her washhouse she often gives them a friendly pat. When they start to rot she will put them into the ground, not the rubbish-tin. They will enrich the soil for her flowers to come. ${ }^{23}$

Was Otti Binswanger über den Ausbruch des Krieges in ihrer Autobiographie schreibt, deckt sich faktisch ziemlich genau mit den Recherchen von Ann Beaglehole und auch mit der fiktionalen Dar-

21 Binswanger, And How Do You Like This Country?, S. 57-58.

22 Ebd., S. 59.

23 Ebd., S. 64. 
stellung von Maurice Gee in Live Bodies (1998). Sie erinnert sich:

Wir wurden unsanft aus der Betäubung geweckt, denn mit einem Schlag hatte sich unser politischer Status im Lande geändert. Bisher waren wir "Refugees", von Hitler Verfolgte gewesen, jetzt wurden wir "Enemy Aliens", zu feindlichen Ausländern. Man behandelte uns glimpflich, man behinderte unsere Tätigkeiten nicht. Einzelfälle wurden in Tribunalen untersucht, die über die allgemeine Situation grobe Ahnungslosigkeit zeigte, über die speziellen Verhältnisse der Einzelnen aber durch den englischen "Intelligence Service" auf das Genaueste informiert waren. Wir wurden in unserer Bewegungsfreiheit wesentlich eingeengt, aber was tat das schon, die Unfreiheit im eigenen Lande wäre wesentlich schwerer zu ertragen gewesen. Wir konnten ungehindert in der Stadt weiter leben und unserer Arbeit nachgehen. Noch ahnten die Neuseeländer nicht, dass dieser Krieg bald auch sie direkt etwas angehen würde. Man schenkte uns Mitleid oder Misstrauen. Die Pensionärin [Binswangers hatten jeweils jemanden zur Untermiete im Haus, um ihr Einkommen etwas zu erhöhen] hatte meine Küche sehr gelobt und sich sehr wohl bei uns gefühlt; am Tage des Kriegsbeginns hatte sie Magenschmerzen. Auf Anraten einer Freundin zog sie sofort aus, Vergiftungsmöglichkeiten fürchtend. ${ }^{24}$

Wie sich nun Otti im folgenden an die Reaktionen ihrer Nachbarn erinnert, entspricht so ziemlich genau dem, was sie in ihrer Erzählung "Turnips" siebzehn Jahre zuvor beschrieben hat:

Der dann folgende Tag war der Tag der Kohlrüben. Wer, der den Ersten Weltkrieg mit ihnen erlebt hat, riecht, schmeckt und verabscheut sie nicht. Die Neuseeländer, nichtsahnend, ziehen sie in ihren Gärten. Jetzt wurden sie geerntet. Die Pfarrerswitwe von gegenüber klingelte bescheiden: "Ich wollte ihnen zeigen, dass wir an sie denken, dass sich nichts in unserer Freundschaft geändert hat." Und sie legte mir ein fünfpfündiges Kohlrübenbaby in den Arm. Kaum hatte ich dies gewichtige Geschenk auf meiner Veranda verstaut, kam ein Rübenbombardemant über den Bretterzaun und zerstörte mein Narzissenbeet. Dahinter stotterte der alte Herr Nachbar: "Es ist wegen des Krieges, der ist doch hart für sie." Der Rübenberg wuchs. Die Nachbarin vom Hintergarten, mir fast unbekannt, legte emsig Kohlrüben über den Zaun ab. "Ich denke, sie können jetzt so etwas gebrauchen, denn sie haben doch Krieg." Die Nachbarin zur Rechten aber hatte ihren alten Fremdenhass aufgewärmt, sie stand lauernd hinter der Gardine und wartete auf das Polizeiauto, das uns abholen würde. 25

24 ,Der Albatros", S. 189.

25 Ebd., S. 190. 
Diese Übereinstimmungen geben Anlaß die Premissen von Otti Binswangers Narration zu untersuchen: was ist Fiktion, was ist erinnerte Wirklichkeit? Sind Fiktion und vorgegebene erinnerte Wirklichkeit austauschbar? Und wenn ja, ist die Annahme hier verifizierbar, daß jeder Text fiktional sei?

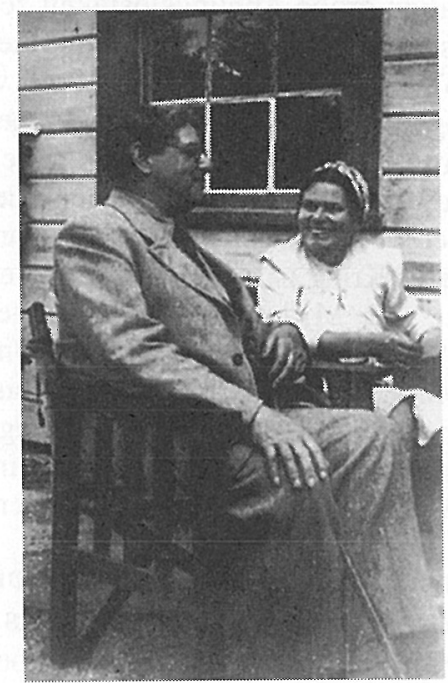

Otti Binswanger und Wolfskehl in Christchurch 1941

(Foto: DLA Marbach)

Sicherlich ist ein erzählerisches Vorgehen nicht neu, das sozialpolitische Stimmungen und Vorurteile, wie sie in der Tagespresse jeweils dokumentiert werden, in ein literarisches Werk einbaut. Was demnach in der literarischen Momentaufnahme "Turnips" über den Anfang des Krieges geschrieben wird, wie die Einstellung insbesondere der "Nachbarn von rechts" den Emigranten, bzw. den "enemy alien" gegenüber konstruiert wird, ist ein bewährtes und legitimes Verfahren im literarischen Diskurs. Wenn jedoch dasselbe Verfahren in einer Narration, die ein Anrecht auf eine erzählte Wirklichkeit erhebt, verwendet wird, dann scheint mir die Fiktionalität beider Gestaltungsformen den Beweis einer Austauschbarkeit zu demonstrieren. Die Grenze zwischen authentischer und konstruierter Erinnerung verwischt sich. Die erinnerte "Wirklichkeit" und die erzählte "Fiktion" werden beide nicht nur durch ein Auswahlverfahren und eine Darstellungsmodalität von Sprache und Mittei- 
lungswertem geprägt, sondern eben auch von einer bewußten Phantasie. Was nämlich die "Nachbarin zur Rechten" denken mag, ist eine Vermutung, die jedoch in der Autobiographie als Tatsache vermittelt wird. Letztenendes konstruiert Otti Binswanger den Gefühls- und Denkvorgang nicht nur einer fiktionalen Gestalt, sondern auch eines erinnerten Menschen. Die Frage für mich ist nicht, ob ein solches Verfahren in der Narration einer erinnerten "Wirklichkeit" legitim sei oder nicht. Was sich vielmehr aus dem Beobachteten ergibt, ist der Hinweis auf das imaginäre und daher auch durchaus kreative Element in der erinnerten Wirklichkeit Otti Binswangers nicht nur in Hinblick auf Neuseeland, sondern auch inbezug auf die Mitspieler ihrer Vergangenheit, unter die auch Karl Wolfskehl gehört.

Otti hat drei zusammenhängende Erinnerungen Karl Wolfskehl gewidmet und ihn an verschiedenen Stellen im „Albatros“ erwähnt. Von den Erinnerungen sind zwei in der Autobiographie enthalten: die erste beschreibt Wolfskehls Besuch bei den Binswangers in Christchurch im Jahre 1941, die zweite den Abschiedsbesuch im Krankenhaus in Auckland im Jahre 1948, als Otti und Paul Neuseeland verließen. Der dritte Text ist eine, mit Hinblick auf ein literaturgeschichtlich interessiertes Publikum, überarbeitete Fassung der beiden ersteren und wird als unveröffentlichtes Manuskript im Deutschen Literaturarchiv Marbach aufbewahrt. Ich werde mich im weiteren auf diesen Text beschränken, zumal er alle wesentlichen Elemente und Details der früher entstandenen Erinnerungen an Wolfskehl beinhaltet und auch, weil er sich spezifisch der "Begegnung mit Karl Wolskehl in Neuseeland" widmet. Zudem versucht dieser Text Wolfskehl in Neuseeland zu situieren. Dabei entsteht das Bild eines Neuseelandes, wie es Otti Binswanger, nicht Wolfskehl erfahren hat. Da jeder wortgewordenen Erfahrung zugleich eine Interpretation zugrundeliegt, ist eben das Bild, das von Wolfskehl und Neuseeland entsteht, in das Interpretationsmuster Otti Binswangers gebettet, wie es ihre Erzählungen und ihre Autobiographie geprägt hatte. Wenig anderes meint Micha Brumlik, wenn er behauptet, daß 'die individuelle Erinnerung eines jeden einzelnen Menschen in einen soziokulturellen Interpretationshorizont eingebettet sei, der seine spezifischen Erinnerungsleistungen prägt und ermöglicht.'26 Das bedeutet nach seiner Meinung,

26 Brumlik, Micha, "Individuelle Erinnerung, kollektive Erinnerung. Psychosoziale Konstitutionsbedingungen des erinnernden Subjekts", in: H. Loewy, B. Moltmann (Hg.), Erlebnis-Gedächtnis-Sinn. Authentische und konstruierte Erinnerung, Frankfurt/New York 1996, S. 34. 
daß menschlicher, individueller oder kollektiver Vergangenheitsbezug, der nichts anderes als ein kultureller Konstruktionsprozeß ist, in stetigem Konflikt zwischen Selbsttäuschung und Selbstbehauptung auf der einen Seite sowie dem Bestreben nach Wahrhaftigkeit auf der anderen Seite steht. Daß die Beziehung von Wahrheit und Wahrhaftigkeit in systematischer Hinsicht komplex ist, leuchtet ein; beim Vergangenheitsbezug, beim Erinnern [...] ist der Wille zur Wahrhaftigkeit eine notwendige Voraussetzung wahrer Erkenntnis. Freilich garantiert auch der gute Wille noch keine wahren Einsichten.

Hinzukommt, daß Otti ihre "Begegnung" mit Karl Wolfskehl erzählerisch bewußt gestaltet. Sie stellt Wolfskehl in den Begegnungskonflikt zwischen ihre europäische Vergangenheit und die neuseeländische Gegenwart. Sie spricht dies selbst aus in der Autobiographie, wo sie notiert:

Ich denke an den Dichter im Exil, Karl Wolfskehl, dem wir jetzt entgegenreisen und der schwer erkrankt in Auckland im Hospital liegt. Er kam in unser Haus in Christchurch, und wir lebten mit ihm einige verklärte Wochen. Entrückt dem neuseeländer Leben, mit dem wir uns täglich neu auseinenderzusetzen hatten, war die Gegenwart Wolfskehls die Bestätigung einer Vergangenheit, die kein Hitler, keine Verbannung anfechten konnten. ${ }^{27}$

In der "Begegnung" steht im Vordergrund eben jener Besuch in Christchurch, der einmal mit 1943 [,Albatros"] und dann mit 1941 ["Begegnung..."] datiert wird. Den Hintergrund bildet Ottis erste Begegnung mit Wolfskehl 1920 auf der Jugendsiedlung Frankenfeld in der Rheinebene, ferner ein kurzer Hinweis auf einen Besuch bei Wolfskehl auf dem Schlösschen Kiechlinsbergen im Kaiserstuhl. Den Rahmen für die erinnerte Gegenwart bildet die kritische Auseinandersetzung Ottis mit ihrem Leben in Neuseeland. Sie schreibt:

Im Jahre 1940 hatten wir begonnen, unser Exil in der Stadt Christchurch auf der Südinsel Neuseelands zu stabilisieren. Der Krieg war weit entfernt, dennoch schlich sich seine unheimliche Gegenwart zu jeder Stunde in unser Leben ein. Zu dankbarem Anerkennen äusserer Sicherheit und leiblichen Wohlbefindens gehörte stets eine Willensanstrengung, als ob man über einen Bretterzaun klettern muss-

27 „Der Albatros“, S. 216. 
te, um dahinter den besonnten Garten zu erblicken. Wir hatten, was damals der unerfüllbare Wunsch von Millionen war: einen grossen alten Bungalow in einem weitläufigen Garten. Aber wir waren Fremde in diesem sauberen, ordentlichen englischen Kolonialland. Alles war vorhanden was man zum Leben brauchte, dennoch erschien die Welt leer. Die Menschen waren freundlich und hilfsbereit, aber ihre Sprache, ihr Lachen und selbst ihre Trauer dienten einem lauwarmen Wohlbehagen, aus dem keiner heraustrat. Was sollte uns zu wünschen übrig bleiben in dieser Zeit des Sterbens und grausamen Leidens in den Regionen, aus denen wir gekommen waren? Zwischen den beiden Spannungspolen: Morgenzeitung und abendliche Nachrichten aus England kroch der Tag in der erzwungenen Zufriedenheit Neuseeländer Lebens dahin, an das sich jetzt anzupassen von uns gefordert wurde. ${ }^{28}$

Und etwas weiter heißt es: "Bei aller menschlichen Güte, von der es viel zu erfahren gibt, schwebt über dem Lande der lauwarme Dunst eines Philisterparadieses."

Für Otti und Paul Binswanger ist Neuseeland ein Land der geistigen Leere, das von ihnen als kulturelle Isolation begriffen wird. Ihre Freude ist groß, als sie erfahren, daß Karl Wolfskehl bereits seit zwei Jahren in Auckland lebt. Sie schreiben an ihn und laden ihn zum Besuch ein, und Wolfskehl folgt dieser Einladung. Das Kernstück von Ottis Text ist zwar der Darstellung dieses Besuches gewidmet, gibt ihr aber die Möglichkeit, auch über ihre früheren Begegnungen mit Wolfskehl zu berichten und somit ein Bild des Dichters zu entwerfen, wie es für ihr eigenes Leben von Bedeutung wurde.

Die erste "Begegnung" von 1920 läßt Wolfskehl "als eine mächtige Männergestalt mit sehr kurzsichtigen Augen" entstehen, der, als plötzlicher Gast in der Jugendsiedlung Frankenfeld erscheinend, "jeden Gegenstand" des "primitiven Haushaltes begeistert abtastete". Sein Name sagte Otti damals nichts, wie sie berichtet, doch, wie sie noch fünfzig Jahre später behauptet, "musste ich mir eingestehen, dass man sich der Ausstrahlung dieses Mannes schwer entziehen konnte." Erinnerungswert ist für sie auch die Beobachtung, daß Wolfskehl mit der Leiterin der Siedlung, Marie Buchhold, einen Darmstädter Dialekt sprach, der als "ihr anderes Deutsch" kultiviert wurde. In diesem Dialekt zitiert Otti Wolfskehls schmeichelhafte Bemerkung über sie als junges Mädchen, allerdings kommentiert sie: "Es muss gesagt sein, dass er; kurzsichtig

28 Ebd., "Begegnung mit Karl Wolfskehl in Neuseeland", Unveröffentlichtes Manuskript, Deutsches Literaturarchiv, Marbach, Bestands/Zugangsnummer: X 71.175 , S. 1. 
und schnell begeistert wie er war, ausserordentlich voreilig im Urteil über Frauenschönheit sein konnte."

\begin{abstract}
"Was wunnerschoins Mädsche!” hörte ich hinter mir. Es lief mir warm den Rücken herunter. Dann war er neben mir, die Siedlungsregentin wurde unwichtig, und wir lernten uns bei dieser kurzen Wanderung in der milden Herbstsonne etwas kennen. Danach hatte ich nur noch indirekt von ihm gehört, und nach Jahren war ich mal für kurze Zeit mit einer Freundin der Wolfskehls bei ihnen zu Gast auf dem Schlösschen Kiechlingsbergen im Kaiserstuhl."'29
\end{abstract}

Im Zusammenhang mit Wolfskehls Besuch in Frankenfeld, bemerkt Otti im „Albatros“, daß sein Interesse an ihr, ihr auf der Siedlung neue Achtung verschafft habe und daß man dachte, sie gehöre nun ganz zu ihnen. "Aber gerade die Begegnung mit dieser Persönlichkeit" hatte sie von "allem Druck freigemacht" und "als die Blätter fielen", reiste sie heim nach Berlin. ${ }^{30}$

Inbezug auf jenen Besuch bei Wolfskehls Jahre später, erinnert sie sich lediglich an etwas Seltsames. Man verlangte nämlich von ihr, "den Namen Binswanger nicht zu erwähnen." Dies sei für sie ein "schwieriges Ansinnen gewesen, erinnert sie sich, da sie im Begriff war, diesen Ketzer zu ehelichen.' Was sich der junge Paul Binswanger, wie Otti berichtet, zuschulden kommen ließ, nämlich daß er bei einer Lesung im George-Kreis (es handelte sich um Georges Dante-Übersetzung) den Raum verlassen hatte, war als Sakrileg befunden worden. Und obwohl die "grollenden Wolken" der Vergangenheit sich "rasch in der Freude gemeinsamer Gegenwart" in der südlichen Hemisphäre auflösten, "wo sonst niemand zu finden war, der noch geistigen Anteil an einem Deutschland hatte, wie es Wolfskehl verkörperte," kommt es wiederum zu einer Verstimmung Wolfskehls bei dem Besuch in Christchurch, die, wenn auch nur auf einen Tag beschränkt, ebenfalls auf Paul Binswangers Weigerung zurückführte, zum "Meister" ähnlich wie Wolfskehl zu stehen. Otti deutet den Vorfall wie folgt:

Die Forderung sollte vielleicht auch eine Besiegelung der Freundschaft bedeuten. Doch es kam nicht dazu. Grollend verließ Wolfskehl das Zimmer und sprach während des übrigen Tages fast nichts mehr mit uns, in der Nacht hörten wir ihn zornig vor sich hinstöhnend über den Rasen stapfen wie ein verwundeter Gott. In dieser Nacht, als wir ihm nicht helfen konnten, wurde uns die Tra-

29 Ebd., S. 2.

30 „Der Albatros“, S. 87. 
gik in Wolfskehls Dichterschicksal deutlich. Warum mußte dieser freie, selbständige Geist sich ein Leben lang George unterstellen, wie es nicht einmal dessen fanatischste Jünger getan und durchgehalten haben, zumal Wolfskehl auch gar kein Männerbündler gewesen ist. 31

Es kommt auch zu einer Lesung Wolfskehls bei den Binswangers. Die vorbereitende Gestaltung der Lesung und ihre Durchführung kommt Otti manieriert vor und sie empfindet die Künstlichkeit der Situation abträglich für den Genuß der Dichtung. Sie macht Margot Rubens mitverantwortlich für die konstruierte Künstlichkeit. In Ottis wertender Erinnerung entsteht das folgende Scenario:

Einige Zeit nach diesem Besuch bei uns verlangte Wolfskehl wieder nach der Gesellschaft Margot Ruben. Sie kam, und es veränderte sich mit der Gemeinschaft der Beiden die Tage unbefangener Heiterkeit. Man steuerte wieder in den Wolkenbau des "Meisters" hinein, wo die Gesetze der "Geisteshohheit" herrschten. Wolfskehl wollte uns Teile seines großen Gedichtes "Mare Nostrum" vorlesen. Die Tatsache, daß einige alte deutsche Freunde um ihn waren, die seine Verse verstehen und würdigen konnten, evozierte den Dichter in ihm. Aber stattdessen geriet ihm der Auftritt zur Pose, die Sinn und Schönheit seines Gedichts kaum aufkommen ließ. Wir mußten in besonderer Reihenfolge auf der Couch sitzen, an der Lampe wurde lange gerückt, Margot Ruben mußte in erlernter Haltung darunter stehen. Die englische Mutter von Klein-Juliet, die kein Wort verstand, mußte auch dabei sein, wurde allerdings durch ihr schreiendes Baby bald vom Krampf der Veranstaltung befreit. Margot Ruben war ein gut gestimmtes Instrument für jene Art Gedichtlesung, wie sie im George-Kreis geübt wurde. Die Monotonie dieses sakralen Worttrompetens mag vielleicht ein Echo im ästhetischen Bewußtsein des Hörers hervorrufen, die Seele aber bleibt taub. Wolfskehls Dichtung hatte wenig mit der Stefan Georges gemein, und doch stellte er sich ganz unter dessen Schatten wie ein Bischof unter den Papst. 32

Ganz anders als ein solcher "Auftritt" gestaltet sich in Ottis Erinnerung der spontane Vortrag des Hiob-Gedichtes als Otti Wolfskehl 1948 in Auckland besuchte. Sie beschreibt die Szene nur kurz: "Eines Abends, als das elektrische Licht versagte, saßen wir bei einer Kerze. Es ergab sich aus der Stimmung guten Einklangs, daß Wolfskehl sich zurücklehnte, die blinden Seheraugen fest geschlos-

31 „Begegnung mit Karl Wolfskehl“, S. 8.

32 Ebd., S. 8. 
sen, und sein Gedicht Hiob sprach. Es war eine große Stunde. Welch eine strömende Quelle war dieser Mann, welch ein Vulkan." Doch zurück zu Wolfskehls Aufenthalt bei Binswangers 1941. Was mich bei Ottis erinnerter "Wirklichkeit" dieses Besuches besonders interessiert, ist, wie Otti eine Unvereinbarkeit der geistigen Welt Europas, deren Repräsentant Wolfskehl (und Paul Binswanger) im Text wird, und des "Philistertums" Neuseelands zum Kontrast werden läßt. Die Frage, die sich mir stellte war: ist diese Unvereinbarkeit, die in dem Text "Begegnung mit Karl Wolfskehl in Neuseeland" einen hervorragenden Stellenwert hat, von Wolfskehl geäußert worden oder ist es Ottis erlebte und erinnerte Welt, in der sie Wolfskehl die Rolle eines Gegenpoles zuweist?

Der oben erwähnte Rahmen, den sie Wolfskehls Besuch in Christchurch gibt, ist nichts weniger als eine sozialkritische Kurzgeschichte der Kolonialisierung Neuseelands. Sie wird an keiner Stelle durch ein Wolfskehl-Zitat unterstützt; eher scheint sich Wolfskehls Einstellung zu Neuseeland als Subtext zu Ottis zu gestalten. Wie kommt ein solcher Effekt zustande?

Etwa in der Mitte der "Begegnung", nachdem Otti eine Kindestaufe beschreibt, bei der Wolfskehl als Pate aktiven Anteil hatte, widmet sie eine lange Passage (etwa ein Sechstel des ganzen Textes) der Beschreibung Neuseelands. Sie leitet die Beschreibung mit der Gestalt Wolfskehls ein, die sie, sich an imaginäre Leser wendend, zur Gegenfigur der Kolonisatoren und ihrer Abkömmlinge werden läßt. Die Entdeckerlust Wolfkehls wird dem Festhalten der Neuseeländer an Traditionen eines anderen Weltteiles gegenübergestellt. Hat sich jedoch Otti nicht selbst das zuschulden kommen lassen, was sie den Neuseeländern britischer Herkunft vorhält? Ist es ihr selbst gelungen, Neuseeland mit neuen Augen zu sehen oder hat sie Land und Leute in die eigenen, d. h. in Europa geformten, soziokulturellen Diskurse eingeordnet?

Ich zitiere einen Teil der oben erwähnten Passage:

Man stelle sich diese ungewöhnliche Erscheinung: den Dichter Karl Wolfskehl, den immer Menschen Suchenden und Menschen Hungrigen in Neuseeland vor, dessen Gesellschaftsstruktur nicht von Abenteurern oder Entdeckern geformt worden war. England hat die Inseln auch niemals wie Australien als Kolonie zur Deportation von Strafgefangenen benutzt. Neuseeland ist in der zweiten Hälfte des vorigen Jahrhunderts von braven englischen, schottischen, irischen Bauern und Kleinbürgern bevölkert worden, die hier ein besseres Vorwärtskommen suchten. In zäher Pionierarbeit haben sie 
sich eine Existenzsicherheit geschaffen, die ihnen "at home" nicht gewährt wurde. Sie wanderten in Gruppen kirchlicher Verbände aus. Keine Entdeckerphantasie hat sich dem Lande aufgeprägt. Pedantische Befolgung der gewohnten Lebensriten beherrschte die Pioniere. Man kochte dieselben Gerichte wie sie Engländer, Schotten und Iren von Väters Zeiten her kannten, man hielt eine puritanische Ordnung des Lebens aufrecht, man handelte den Eingeborenen, den Maoris, das Land ab, nachdem ihnen schon einige Jahrzehnte vorher in blutiger Unterwerfung das Rückgrat gebrochen worden war.

Heute sind auch die Maoris Neuseeländer. Man behandelt sie freundlich und es stehen ihnen alle Möglichkeiten des Fortkommens offen. Der allgemeine Wohlstand des Landes ist gesichert. Englandtreu wie kein anderes der Commonwealthländer genießen die Neuseeländer auch des Mutterlandes Treue in Ex- und Importgarantien für Wolle, Fleisch und Butter. Die Wellblech bedeckten einstöckigen Holzhäuser stehen in Blumenbeeten und wohlgepflegten Rasenflächen. Das Auto in der Garage, der Hammel im Ofen, das Huhn im Topfe, und in den Küchen und Wohnräumen häufen sich die Konsumgüter. Aber man ist sehr weit von Europa entfernt und erlebte darum nur sehr am Rande die gesellschaftlichen Umordnungen, die sich nach dem ersten Weltkrieg vollzogen. Alle bürgerlichen Sicherheiten, inzwischen so fraglichen Wertes, sind für Neuseeland die unumstößlichen Rückendeckungen geblieben. Bei aller menschlichen Güte, von der es viel zu erfahren gibt, schwebt über dem Lande der lauwarme Dunst eines Philisterparadieses. ${ }^{33}$

Diese Sicht überträgt Otti Binswanger in die Interpretation von Wolfskehls veränderter Erscheinung, wenn sie behauptet, daß "nicht das Alter allein" ihn "ruhiger und milder gemacht hätte." Vielmehr, meint sie, sei es darauf zurückzuführen, daß sich Wolfskehl "dem gemäßigten menschlichen Klima" von Neuseeland auzupassen suchte. Und er "dankte aus vollem Herzen", schreibt sie, "den vielen Freundlichkeiten, die ihm erwiesen wurden. Immer war seine Freude groß, wenn er neue Menschen kennenlernte, besonders wenn sie ein Organ für Dichtung hatten." Sie fügt hinzu: "Es wäre falsch zu sagen, daß Wolfskehl seine Tage allein auf einsamen Felsen in Neuseeland vertrauert hätte. Die letzten Jahre haben ihn auch nicht verstummen lassen". Und was Wolfskehls in Neuseeland geschriebenes Werk anlangt, kommentiert Otti Binswanger wertend: "vielleicht sind seine besten Dichtungen hier entstanden." Sogar auf sein körperliches Wohlbefinden dehnt sie ihre wertende Sicht aus. Sie schreibt: "Auch körperlich schien ihm

33 Ebd., S. 6. 
der Aufenthalt in Neuseeland gut zu tun."

In diesem Zusammenhang wären auch die Beobachtungen zu erwähnen, die Wolfskehls Verhältnis zur Natur gewidmet sind. Otti fällt vorerst die Aufgeschlossenheit und Offenheit Wolfskehls für alles Neue auf. Sie schreibt: "So betrat er jetzt unsern Rasen, unser Haus und freute sich an vielem, das dieser neue Aufenthaltsort bot." Er ging auch gerne aus, die Umgebung zu erforschen, und Otti beschreibt einen Sonntag Nachmittag, als sie mit Wolfskehl im Hagley Park in Christchurch spazieren ging. "Als unverbesserliche Europäer", kommentiert sie, "träumten wir von einem Tee im Gartenpavillon, und wurden natürlich von den puritanisch geschlossenen Rolläden bitter enttäuscht." Otti entschließt sich daher, als Trost sozusagen, Wolfskehl etwas Besonderes zu zeigen. Sie schreibt:

So hatte ich die Idee, Karl Wolfskehl einen kleinen, noch erhaltenen Flecken Urwald zu zeigen, "Deans Bush", der in der Richtung unseres Weges lag. Ich hatte jedoch die Entfernung unterschätzt und Wolfskehl taten bald die Füße weh, er wollte wie ein kleiner Junge nicht weiter gehen. Die Hälfte des Weges lag aber schon hinter uns und ich mochte den Ausflug nicht als Mißerfolg enden lassen. Mein Begleiter litt, wie eben nur Männer unter Wehwehchen zu leiden verstehen; er beschimpfte mich fürchterlich wegen meiner schlechten Schätzung. Mit allerlei Tricks brachte ich ihn dann doch zu dem kleinen Naturwunder: Feuchter, überwucherter Boden, wildes Geranke, hohe Urwaldbäume und das fröhlich Gezwitscher angstloser Waldvögel. Die einzige Bank stand noch da und war leer. Sobald Wolfskehl saß und in den Bann des Genius Loci geraten war, war er ein anderer, dankte mir heiß, ihn an diesen außerordentlichen Platz geführt zu haben, der noch die magischen Kräfte wirklichen Urwalds ausströmte. Er sog die Luft, den Duft tief in sich hinein, befühlte Baum und Strauch, roch an Gräsern und entzückte sich an den Vogelstimmen. ${ }^{34}$

Auch bei ihrem Besuch in Auckland 1948 fällt Otti Wolfskehls tiefe Verbundenheit mit der Natur auf. Sie erinnert sich: "Seine unmittelbare Beziehung zur Natur beglüickte mich besonders. So wanderten wir einmal eine von Eucalyptusbäumen gesäumte Straße entlang. Sie standen in Blüte. Ich griff über mich und brach einen Zweig. An den graugrünen harten Blättersicheln standen die kleinen purpurnen Puscheln wohl die zartesten aller Blüten, die an diesem Baum des trockensten, ärmsten Bodens aufgehen. Der

34 Ebd., S. 5. 
kleine Zweig wurde in diesem Augenblick für Wolfskehl zu großer Wichtigkeit: er betastete die Blüte, hielt sie vor das Gesicht und erkannte sie bis in ihre intimste Struktur und ihren Ausdruck." 35 All diese Zitate formen einen Gegendiskurs zu Ottis eigenem, der, wie erwähnt, Wolfskehls Gestalt als Kontrast zu einer kulturellen und geistigen Öde, wie Neuseeland es für Otti gewesen war, benutzen will. Zitat und interpretierende Absicht stehen in kreativer Spannung zueinander, die Einblick in eine Offenheit und Vorurteilslosigkeit des Dichters der fremden soziokulturellen Umgebung und Natur gegenüber gewährt. Man könnte sogar folgern, daß diese Offenheit und Vorurteilslosigkeit, die Otti im Zusammenhang mit Wolfskehl 'Entdeckerlust' nannte, sich in ihrer erinnerten Darstellung Wolfskehls in dessen Einstellung zum eigenen Tod behauptet. Binswangers hatten geplant, Neuseeland zusammen mit Wolfskehl zu verlassen. Doch als es, wie Otti schreibt, "im Jahre 1948 endlich so weit war, war es für ihn schon zu spät, er wußte, daß sich der Kreis seines Lebens schloß, daß es keinen neuen Anfang mehr für ihn geben würde." Und sie zitiert bei der Beschreibung der letzten Begegnung im Krankenhaus in Auckland, wo Wolfskehl sein Leben beendete, einen Spruch von früher, als er vom Sterben sprach: "Warum soll ich mich fürchten, von einem Raum in den anderen $\mathrm{zu}$ treten?"36

35 Ebd., S. 10.

36 Ebd., S. 10. 